\title{
REVIEW
}

\section{Clinical review: Guyton - the role of mean circulatory filling pressure and right atrial pressure in controlling cardiac output}

\author{
William R Henderson*1, Donald E G Griesdale', Keith R Walley² and A William Sheel ${ }^{3}$
}

\begin{abstract}
Arthur Guyton's concepts of the determinative role of right heart filling in cardiac output continue to be controversial. This paper reviews his seminal experiments in detail and clarifies the often confusing concepts underpinning his model. One primary criticism of Guyton's model is that the parameters describing venous return had not been measured in a functioning cardiovascular system in humans. Thus, concerns have been expressed in regard to the ability of Guyton's simplistic model, with few parameters, to model the complex human circulation. Further concerns have been raised in regard to the artificial experimental preparations that Guyton used. Recently reported measurements in humans support Guyton's theoretical and animal work.
\end{abstract}

\section{Introduction}

The support of blood flow is one of the central goals of clinical medicine, and the understanding of the regulation of blood flow is the sine qua non of cardiac physiology. Building on the foundational work of Frank and Starling, Arthur Guyton proposed that characteristics of the venous circulation were of fundamental importance in the regulation of cardiac output and thus blood flow. However, several authors have raised strong objections to Guyton's model, and more than 50 years after the publication of his model, there is still debate about whether Guyton's ideas present a viable model of cardiac control or whether several fundamental misjudgments lie at the core of Guyton's conclusions [1-4].

\footnotetext{
*Correspondence: william.henderson@vch.ca

${ }^{1}$ Critical Care Medicine, Vancouver General Hospital, ICU, JPPN, 2nd Floor, Room 2438, 899 West 12th Avenue, Vancouver, BC, V5Z 1M9, Canada Full list of author information is available at the end of the article
}

\section{A brief history of cardiac output}

Traditionally, the heart's accepted role has been that it not only provides the driving force for blood flow but also determines the total blood flow [5-7]. Simply stated, cardiac output is the product of stroke volume and heart rate. In this view, all pressures in the heart and circulatory system (for example, those measured in the large veins, in the cardiac chambers, and in the arteries) are derivatives of the force generated by the heart rather than independent variables that might have an influence on the heart's function and thus cardiac output.

At the end of the 19th century, Frank [8] found that ventricular contractility was increased if the ventricle was stretched prior to contraction. Building on this observation, Starling and colleagues $[9,10]$ found that increasing venous return increased stroke volume. We therefore term the ability of the heart to change its force of contraction (and stroke volume) in response to changes in venous return the Frank-Starling mechanism.

The ventricle does not operate on a single FrankStarling curve. Any heart may operate on a family of curves, each of which is defined by the afterload, inotropic state, and diastolic compliance of the heart. Changes in venous return cause the ventricle to move along a single Frank-Starling curve that is defined by the existing conditions of afterload and inotropy and diastolic compliance.

\section{Guyton's observations and model}

Guyton felt that three factors were central in the determination of cardiac output: the pumping function of the heart, the resistance to blood flow through the peripheral circulation, and the degree of filling of the circulatory system with blood [11].

The heart's permissive role in the determination of cardiac output

If, Guyton reasoned, cardiac output is governed solely by heart function, then changing either heart rate or the heart's pumping ability should change cardiac output [12]. Extending the observations of Brauwald and 
colleagues [13] that cardiac output was largely unaffected by heart rate when subjects were electrically paced, Guyton electrically paced the hearts of dogs that had a surgically created arteriovenous fistula between the aorta and the inferior vena cava [14]. Prior to the opening of this fistula, changes in heart rate had no effect on cardiac output. However, when the fistula was opened (causing increased preload as evidenced by high right atrial pressure $\left[\mathrm{P}_{\mathrm{RA}}\right]$ values), cardiac output increased in proportion to heart rate changes. The advent of extracorporeal circuits allowed Guyton to question whether the intrinsic pumping ability or contractility of the heart was the sole determinant of cardiac output [15]. When the pump speed of the extracorporeal circuit was increased, cardiac output did not increase significantly. However, by increasing the pump speed enough to lower $\mathrm{P}_{\mathrm{RA}}$ to zero, the thoracic veins collapsed, thereby limiting flow. From these observations, Guyton concluded that at steady state the heart played a permissive role. In Guyton's model, the heart will pump as much blood as is presented to it, within the limits of intrinsic contractility and heart rate. To demonstrate this, Guyton and colleagues $[16,17]$ increased the blood volume of dogs by $30 \%$ to $40 \%$ over several minutes by venous transfusion. In all animals, cardiac output initially doubled and remained approximately $20 \%$ above pre-transfusion levels. Guyton had shown that, independently of heart rate, intravenous volume increases profoundly affected cardiac output.

\section{Factors peripheral to the heart determine cardiac output}

Guyton felt that, in addition to heart function, characteristics of the peripheral circulation (and particularly the venous circulation) played a central role in determining cardiac output. The two key descriptors of the peripheral circulation that Guyton felt were critical to the understanding of cardiac output were $\mathrm{P}_{\mathrm{RA}}$ and 'mean circulatory filling pressure' (MCFP).

$\mathrm{P}_{\mathrm{RA}}$ as a determinant of cardiac output was not a novel concept. Demonstrating this relationship in intact subjects is difficult because of the numerous compensatory events that occur in response to any change in $\mathrm{P}_{\mathrm{RA}}$ or cardiac output. To overcome this, Guyton performed rapid transfusions of anesthetized dogs that had previously damaged myocardium or that were receiving epinephrine infusions. $\mathrm{P}_{\mathrm{RA}}$ can also be seen as an impediment to the flow of venous blood into the right atrium. If $\mathrm{P}_{\mathrm{RA}}$ impedes flow, what drives flow? To answer this, Guyton proposed a novel concept: mean circulatory filling pressure [18].

\section{Mean circulatory filling pressure}

Whereas Weber coined the term 'statischer Fullungsdruck' ('static filling pressure'), Guyton made 'mean circulatory filling pressure' a central component of his model [19]. Earlier authors had recognized some of the concepts contained within it [19-21]. MCFP represents the average integrated pressure throughout the circulatory system. It can be measured by stopping blood flow and allowing the pressures throughout the circulatory system to reach equilibrium. It may be thought of as a measure of the elastic recoil potential stored in the walls of the entire circulatory system (including the heart and pulmonary circulation). As such, it is a function of the volume of fluid within the system and the capacitance of the system. As more fluid enters the circulatory system (such as during transfusion), the MCFP increases because the elastic energy within the system increases. In contrast, increasing the capacitance of the system (due to vessel wall relaxation) will decrease MCFP.

Mean systemic filling pressure (MSFP), though often confused with MCFP and often similar in value, is different. MSFP represents the pressure generated by elastic recoil in the systemic circulation during a no-flow state. MSFP can be affected by the distribution of flow and volume prior to stopping circulation (for example, it will be higher in procedures that obstruct right heart inflow prior to measurement than in those that do not) [22].

MCFP, and not the arterial pressure generated by the heart, is what drives the flow of blood toward the right atrium in Guyton's model. It is MCFP that overcomes venous resistance and $P_{R A}$. The net driving pressure for venous return to the heart was described by the difference between MCFP and $P_{R A}$. This concept has often been taught by using a bathtub analogy originally proposed by Magder and colleagues [23,24].

The rate at which a bathtub empties is a function of the height of water above the drain in the tub and the tub drain's characteristics, which include the resistance to flow and the pressure downstream of the drain. In this analogy, the inflow of water from the tap may be thought of as arterial pressure and flow, the level of water in the tub as MCFP (the elastic recoil in the system), and the drain as the venous resistance to flow and $P_{R A}$. The 'force' of flow from the tap (arterial pressure) into the tub does not directly affect drainage beyond increasing the level of water in the tub. It is the elastic recoil of the systemic vessels (both veins and arteries) that determines flow in the systemic circulation. If the downstream pressure is the same as the pressure in the tub, the tub will not empty. Analogously, when the pressure downstream $\left(\mathrm{P}_{\mathrm{RA}}\right)$ is equal to MCFP, there is no flow; flow can occur only when MCFP is greater than $P_{R A}$.

\section{What controls mean circulatory filling pressure?}

If venous return to the heart and thus cardiac output are dependent on MCFP, the question then is, what controls MCFP? It is not explicitly stated in Guyton's early work 
but it became obvious in his later writing [12] that he believed that resistance and capacitance at the organ and muscle levels were the central issue. During periods of increased oxygen demand (exercise, fever, increased metabolism), the affected organs would release unidentified mediators that cause vasodilation in their local vascular beds.

This concept is similar to both Krogh's and Caldini's two-compartment model of venous return [25-28], wherein the systemic circulation consists of (at least) two parallel compartments with different venous time constants. In the short time constant, blood moves quickly from the arterial system through the vascular bed into the venous circulation with little resistance. In the long time constant, compartment transit through the vascular bed takes longer, and less blood is available to fill the venous circulation. An increase in the proportion of blood flow through the fast time constant compartment would cause a net decrease in the total transit time of blood flow into the venous system. Thus, the redistribution of cardiac output to the short time constant circulation from the long time constant circulation during exercise or illness might increase venous return and thus cardiac output [29].

\section{Venous return and cardiac output depend on mean circulatory filling pressure}

To investigate the effect of MCFP and $\mathrm{P}_{\mathrm{RA}}$ on venous return, Guyton and colleagues [15] cannulated the right atria of 15 anesthetized dogs. This cannula was attached to a motorized pump, and the output cannula was placed in the proximal aorta. MCFP was varied by altering the amount of fluid in the circulatory system, and $P_{R A}$ was altered by changing the pump speed. To alter $\mathrm{P}_{R A}$, Guyton adjusted the height of the Starling resistor relative to heart level, keeping the resistor in a state of partial collapse. $\mathrm{P}_{\mathrm{RA}}$ was therefore established by the length of the hydrostatic column between the level of the resistor and the level of the atrium. MCFP was determined by stopping the circulation (pump) and measuring pressures once arterial and venous pressures had come to equilibrium. Using this model, Guyton was able to describe the first of his now famous 'venous return curves' (Figure 1). Guyton felt this demonstrated that the rate of venous return is dependent on both the characteristics of the peripheral circulation (MCFP) and the impedance to venous return $\left(\mathrm{P}_{\mathrm{RA}}\right)$.

\section{Clinical utility of Guyton's model}

Guyton recognized that, during steady-state physiology, venous return must equal cardiac output and that one therefore could relate cardiac output directly to the determinants of venous return. This was achieved by graphically superimposing venous return curves on

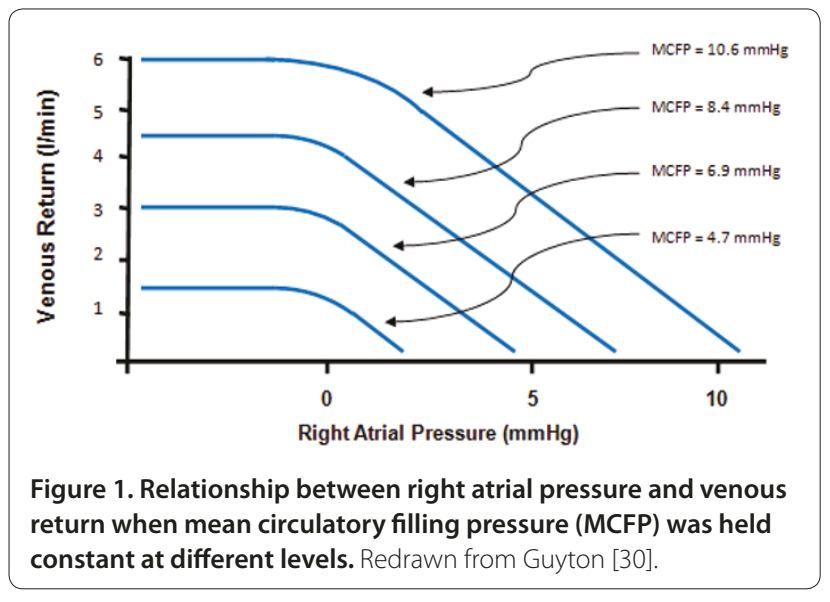

cardiac function curves. By combining cardiac function curves with venous return curves (Figure 1) in a single diagram (Figure 2), Guyton could display the interrelationships between $P_{R A}$, MCFP, venous return, and cardiac output. The power of this combined model of cardiac output and venous return is perhaps best demonstrated through the evaluation of several clinical scenarios.

\section{The normal state and acute volume transfusion}

In Figure 2, the intersection of the cardiac output curve and the venous return curve of a normal subject occurs at a single intercept: point $A$, where $P_{R A}$ is approximately zero. With acute transfusion, MCFP will increase. We can see the effect that this will have on cardiac output by looking at the intercept of this new venous return curve ('increased MCFP') with the normal cardiac output curve: point $\mathrm{B}$, which has a higher associated cardiac output. Thus, the model has predicted that increasing MCFP by volume infusion will increase cardiac output without an alteration in intrinsic cardiac functionexactly as Guyton's experiments demonstrated [30].

\section{Acute hemorrhage or vasodilatory shock}

In normal subjects, decreasing intravascular volume will decrease MCFP and generate a new venous return curve ('decreased MCFP' in Figure 2). If there is no change in cardiac function, the new intercept (point $\mathrm{C}$ ) predicts a lower cardiac output. Likewise, if MCFP is changed by increasing capacitance (as in sepsis) rather than by decreasing volume, the same result occurs. In both situations, the solution is suggested by Guyton's diagram: by increasing MCFP (by infusing volume or decreasing capacitance with vasoconstrictors), the patient can be returned to normal cardiac output (point A).

Recent research and clinical guidelines have promoted early goal-directed therapy (EGDT) as a useful paradigm for the resuscitation of patients with severe sepsis or 


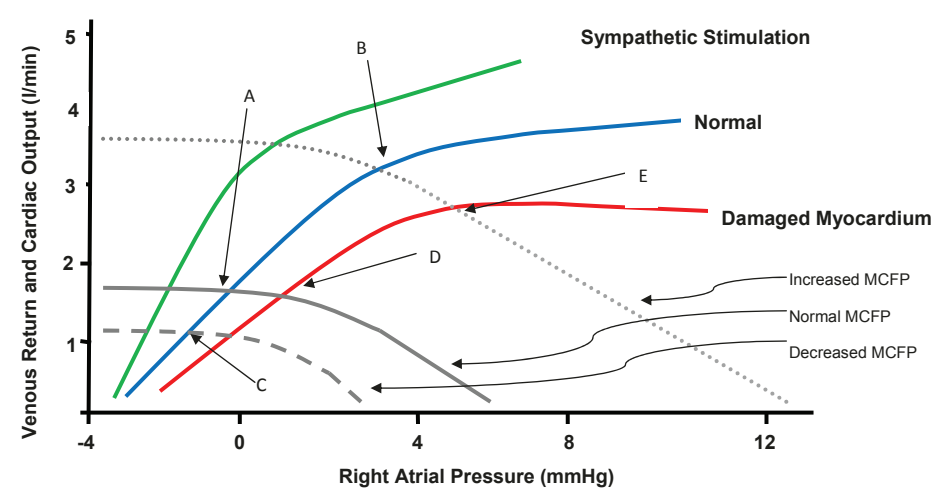

Figure 2. Cardiac function curves can be superimposed on venous return curves. Cardiac function curves (green, blue, and red lines) depict the heart function under different conditions. Venous return curves (grey lines) represent normal, decreased, and increased values of mean circulatory filling pressure (MCFP). Redrawn and modified from Guyton [30].

septic shock. A central strategy in EGDT is to maintain central venous pressure (CVP) between 8 and $12 \mathrm{~mm} \mathrm{Hg}-$ purportedly to improve cardiac output [31]. Despite the widespread belief that CVP reflects the adequacy of cardiac preload in critically ill patients, there is a large body of evidence suggesting that the relationship between CVP and cardiac output is tenuous [32]. Rather, CVP represents the interaction between pump function and venous return and can give meaningful information about volume status if some measurement of cardiac function is known [33].

\section{Heart failure}

With decreasing cardiac function in a normal subject (as after myocardial infarction), Guyton's model predicts that if MCFP is unchanged, cardiac output will decrease (point D). Interestingly, the model also predicts that $\mathrm{P}_{\mathrm{RA}}$ will increase. Again, solutions are suggested by the model. Either increasing cardiac function (via inotropic medications or mechanical support devices) or increasing MCFP (via volume infusion or venoconstrictors) will return the subject to a normal cardiac output: points A and E, respectively. However, the model predicts that this will occur at different values of $P_{R A}$. The clinical examples above demonstrate the power of Guyton's model of cardiac output. The ability to clearly explain and predict the outcomes of manipulating the various factors of vascular and cardiac function has led to the wide adoption and acceptance of Guyton's model.

\section{Criticisms of Guyton's model}

Criticisms of the model fall into one of two main categories: (a) that the experiments upon which Guyton's conclusions were based do not reflect physiologic reality or (b) that the conclusions drawn are flawed. The central criticism of Guyton's experiments involves the physical experimental model used to demonstrate the relationship between $P_{R A}$, MCFP, and venous return and changes in cardiac output [1,5]. In Guyton's original paper, this is not described. In subsequent publications $[15,30,34]$, it appears that the researchers adjusted pump output to control $\mathrm{P}_{\mathrm{RA}}$ 'by increasing or decreasing the minute capacity of the pump' [30]. One interpretation [5] of these statements is that the researchers actively adjusted the pump after each change in elevation of the Starling resistor to obtain the desired state of partial collapse of the resistor tubing. If this is so, Brengelmann [1] suggests, it raises substantial questions about the model used. A central argument of Guyton's model is that $P_{R A}$ controls cardiac output. If the researchers changed the pump speed based on the height of the Starling resistor, this experimental model operated in a fashion that is opposite to Guyton's theory and it was actually pump speed (that is, cardiac output) that controlled $\mathrm{P}_{\mathrm{RA}}$.

It is, however, difficult to imagine how else to test this model, proponents of Guyton's model might suggest. The model of Caldini and colleagues [26] identifies three partial differentials needed to resolve the authors' equation for the vasculature: cardiac output, $\mathrm{P}_{\mathrm{RA}}$, and stressed volume. It is necessary to hold one of these variables constant to assess the relationship between the other two. Thus, to assess the effect of volume on cardiac output/venous return, $\mathrm{P}_{\mathrm{RA}}$ must be held constant. Within the experimental model used by Guyton, this required that pump speed change.

The other broad criticism of Guyton's model is that it contains misinterpretations of the original experimental data, specifically that data generated from several static measurements have been interpreted as describing the dynamics of venous return and cardiac output.

Critics of venous return curves argue that the technique of representing the data as curves (rather than as a series of discrete, static points) encourages the belief that there are dependent relationships between $P_{R A}$, 
MCFP, and venous return. In one critic's words, 'in that model ... venous return is driven through a fixed hydraulic resistor connected between a pressure source fixed at MCFP and an independently variable "back pressure" $P_{R A} \ldots$ this practice of taking the components of the model as having actual counterparts in the vasculature confuses a mathematical abstraction with reality' [1]. Additionally, Guyton did not clarify how his model might explain what occurs when cardiac output is on the flat portion of the cardiac function curve. In this situation, both $\mathrm{P}_{\mathrm{RA}}$ and MCFP may rise without a change in cardiac output.

\section{Recent studies}

Until recently, the measurement of venous return curves in a functioning human cardiovascular system was elusive. Schipke and colleagues [35] measured MCFP in humans during cardiac arrest-an artificial circumstance. Jellinek and colleagues [36] studied the effect of increases in airway pressure on venous return in patients with iatrogenic ventricular fibrillation during pacemaker implantation. Increases in airway pressure decreased cardiac output but did cause equal increases in $P_{R A}$ and MCFP. Thus, decreases in cardiac output did not appear to be from decreases in the venous pressure gradient but rather from increases in resistance to venous return.

In the past year, Maas and colleagues [37] reported the first measurements of human venous return curves in intact human circulation. The authors estimated $P_{R A}$ by measuring CVP and estimated venous return by measuring cardiac output using pulse contour analysis. During apnea at a variety of inspiratory pressures on mechanical ventilation, Maas and colleagues constructed venous return curves in individual patients. In a confirmation of Guyton's theoretical and animal work, the authors found surprisingly linear venous return curves and found that MCFP changed as predicted by Guyton. However, the estimate of MCFP in this study was $18.8 \mathrm{~mm} \mathrm{Hg}$ in euvolemic supine patients. This is considerably higher than previous estimates [22,23,35]. It is unclear whether this may have been due to the experimental protocol or to patient characteristics (the subjects were post-cardiac surgery patients and conceivably had high right-sided cardiac filling pressures due to chronic or post-operative decreased ventricular function). Despite these concerns, the work by Maas and colleagues [37] lends considerable 'real world' support to the use of Guyton's model in intact human subjects.

\section{Conclusions}

Arthur Guyton's model of cardiac output governed by the relationship between $\mathrm{P}_{\mathrm{RA}}$ values and the MCFP has simultaneously confused and clarified thinking about cardiac physiology for half a century. That his insights continue to inform debate is a testament to the flexibility and utility of his model. However, it is critical to remember that his model is simply that: an approximation of reality, not reality itself. Despite recent human evidence that supports Guyton's model, several valid concerns about the experimental preparations from which his concepts derive and about the interpretation of his data exist.

\section{Abbreviations}

CVP, central venous pressure; EGDT, early goal-directed therapy; MCFP, mean circulatory filling pressure; MSFP, mean systemic filling pressure; $P_{R A^{\prime}}$, right atrial pressure.

Competing interests

The authors declare that they have no competing interests.

\section{Authors' contributions}

WRH helped write and edit the manuscript and provided Figures 1 and 2 . DEGG, KRW, and AWS helped write and edit the manuscript. All authors read and approved the final manuscript.

\section{Author details}

'Critical Care Medicine, Vancouver General Hospital, ICU, JPPN, 2nd Floor, Room 2438, 899 West 12th Avenue, Vancouver, BC, V5Z 1M9, Canada. ${ }^{2}$ Heart + Lung Institute, St. Paul's Hospital, Room 166, 1081 Burrard Street, Vancouver, BC, V6Z 1Y6, Canada. ${ }^{3}$ Health and Integrative Physiology Lab, Osborne Ctr Unit II - Room 208, School of Human Kinetics, 210 - 6081 University Boulevard, Vancouver, BC, V6T 1Z1, Canada.

Published: 1 December 2010

\section{References}

1. Brengelmann $G L: A$ critical analysis of the view that right atrial pressure determines venous return. J Appl Physio/ 2003, 94:849-859.

2. Magder S: Point: the classical Guyton view that mean systemic pressure, right atrial pressure, and venous resistance govern venous return is/is not correct. J Appl Physiol 2006, 101:1523-1525.

3. Jayant $A$ : What is simple is perhaps not always the truth. Anesthesiology 2008, 109:933-934

4. Pinsky MR, Permutt S, Wang YL, Wang WK, Baker RD, Rothe C, Mitzner W: Letters to editor. J Appl Physiol 2006, 101:1528-1530.

5. Levy MN: The cardiac and vascular factors that determine systemic blood flow. Circ Res 1979, 44:739-747.

6. Rowell LB: Human Cardiovascular Control. New York: Oxford University Press; 1993.

7. Rushmer RF, Smith OA: Cardiac control. Physiol Rev 1959, 39:41-68.

8. Frank O: On the dynamics of cardiac muscle [in German]. J Biol 1895, 32:370-447.

9. Patterson SW, Starling EH: On the mechanical factors which determine the output of the ventricles. J Physiol (Lond) 1914, 48:357-379.

10. Patterson SW, Piper $H$, Starling EH: The regulation of the ventricles. J Physiol (Lond) 1914, 48:465-513.

11. Guyton AC: Regulation of cardiac output. Anesthesiology 1968, 29:314-326.

12. Guyton AC: The relationship of cardiac output and arterial pressure control. Circulation 1981, 64:1079-1088.

13. Ross J Jr., Linhart JW, Brauwald E: Effects of changing heart rate in man by electrical stimulation of the right atrium. Circulation 1965, 32:549-558.

14. Cowley AW, Guyton AC: Heart rate as a determinant of cardiac output in dogs with arteriovenous fistula. Am J Cardiol 1971, 28:321-325.

15. Guyton AC, Lindsey AW, Abernathy B, Richardson T: Venous return at various right atrial pressures and the normal venous return curve. Am J Physiol 1957, 189:609-615.

16. Dobbs WA, Prather JW, Guyton AC: Relative importance of nervous control of cardiac output and arterial pressure. Am J Cardio/ 1971, 27:507-512.

17. Prather JW, Taylor AE, Guyton AC: Effect of blood volume, mean circulatory pressure, and stress relaxation on cardiac output. Am J Physiol 1969, 216:467-472.

18. Guyton AC, Lindsey AW, Kaufmann BN: Effect of mean circulatory filling 
pressure and other peripheral circulatory factors on cardiac output. Am J Physiol 1955, 180:463-468.

19. Weber $\mathrm{EH}$ : On the application of wave theory to the theory of the circulation of the blood and in particular to the pulse [in German]. Arch Anat Physiol 1851, 18:497-501.

20. Bolton C: The experimental production of uncompensated heart disease with especial reference to the pathology of dropsy. J Path Bact 1903, 9:67-73.

21. Starr I: The role of the static blood pressure in abnormal increments of venous pressure, especially in heart failure. Part II: clinical and experimental studies. Am J Med Sci 1940, 199:40-55.

22. Magder S, Veerassamy S, Bates JHT: A further analysis of why pulmonary venous pressure rises after the onset of LV dysfunction. J App/ Physio/ 2009, 106:81-90.

23. Magder S, De Varennes B: Clinical death and the measurement of stressed vascular volume. Crit Care Med 1998, 26:1061-1064.

24. Magder S, Scharf SM: Venous return. In Respiratory-Circulatory Interactions in Health and Disease. New York: Marcel Dekker; 2001.

25. Krogh A: Regulation of the supply of blood to the right heart (with a description of a new circulation model). Scand Arch Physiol 1912, 27:227-248

26. Caldini P, Permutt S, Waddell JA, Riley RL: Effect of epinephrine on pressure, flow, and volume relationships in the systemic circulation of dogs. Circ Res 1974, 34:606-623.

27. Mitzner W, Goldberg H: Effects of epinephrine on the resistive and compliant properties of the canine vasculature. J App/ Physio/ 1975 39:272-280.

28. Green JF: Mechanisms of action of isoproterenol on venous return. Am J Physiol 1977, 232:H152-H156.

29. Magder $\mathrm{S}$ : Theoretical analysis of the noncardiac limits to maximum exercise. Can J Physiol Pharmacol 2002, 80:971-979.
30. Guyton AC: Determination of cardiac output by equating venous return curves with cardiac response curves. Physiol Rev 1955, 35:123-129.

31. Dellinger RP, Levy MM, Carlet JM, Bion J, Parker MM, Jaeschke R, Reinhart K, Angus DC, Brun-Buisson C, Beale R, Calandra T, Dhainaut JF, Gerlach H, Harvey M, Marini JJ, Marshall J, Ranieri M, Ramsay G, Sevransky J, Thompson BT, Townsend S, Vender JS, Zimmerman JL, Vincent JL: Surviving Sepsis Campaign: international guidelines for management of severe sepsis and septic shock. Intensive Care Med 2008, 34:17-60

32. Marik PE, Baram M, Vahid B: Does central venous pressure predict fluid responsiveness?: a systematic review of the literature and the tale of seven mares. Chest 2008, 134:172-178.

33. Magder S: Central venous pressure: a useful but not so simple measurement. Crit Care Med 2006, 34:2224-2227.

34. Guyton $\mathrm{AC}$, Jones $\mathrm{CE}$, Coleman TG: Effect of right atrial pressure on venous return - the normal venous return curve. In Circulatory Physiology: Cardiac Output and Its Regulation. 2nd ed. Philadelphia, PA: Saunders; 1973.

35. Schipke JD, Heusch G, Sanii AP, Gams E, Winter J: Static filling pressure in patients during induced ventricular fibrillation. Am J Physiol Heart Circ Physiol 2003, 285: $\mathrm{H} 2510-2515$

36. Jellinek H, Krenn H, Oczenski W, Veit F, Schwarz S, Fitzgerald RD: Influence of positive airway pressure on the pressure gradient for venous return in humans. J App/ Physio/ 2000, 88:926-932.

37. Maas JJ, Geerts BF, van den Berg PCM, Pinsky MR, Jansen JRC: Assessment of venous return curve and mean systemic filling pressure in postoperative cardiac surgery patients. Crit Care Med 2009, 37:912-918.

doi:10.1186/cc9247

Cite this article as: Henderson WR, et al:: Guyton: the role of mean circulatory filling pressure and right atrial pressure in controlling cardiac output. Critical Care 2010, 14:243. 